\title{
A Game-theoretic Approach for Efficient Clustering in Wireless Sensor Networks
}

\author{
Zhanyang $\mathrm{Xu}^{1,2}$, Yue Yin ${ }^{1,2}$, Jin Wang ${ }^{1,2}$ and Jeong-Uk Kim ${ }^{3}$ \\ ${ }^{1}$ School of Computer and Software, Nanjing University of Information Science \& \\ Technology, Nanjing 210044, China \\ 2 Jiangsu Engineering Center of Network Monitoring, Nanjing University of \\ Information Science \& Technology, 210044, China \\ ${ }^{3}$ Department of Energy Grid, Sangmyung University, Seoul 110-743, Korea
}

\begin{abstract}
In wireless sensor networks, clustering divides network into clusters and makes cluster heads $(\mathrm{CHs})$ responsible for data aggregation. CHs play a significant role in such topology and focus should be fixed on the CH selection. Due to the constraints on ayailable resources, however, a sensor node is likely to be selfish and refuse to serve as a CH. Based on game theory, this paper models the problem and discusses the condition of Nash Equilibrium. Moreover, in case of disconnection between a CA and the sick data replication is adopted. We set candidate CHs that replicate data in the original CHs respectively under the scenario of a second price sealed auction. Simulation results show that nodes have tendency to cooperate due to the reduction in delay and loss ate Moover the throughput of the sink can still be guaranteed if any $\mathrm{CH}$ fails to work.
\end{abstract}

Keywords: Wireless sensor networks; clustering; game theory; data replication; second price sealed auction

\section{Introduction}

Wireless sensor networks (WSNs) [1] consist of hundreds or thousands of sensor nodes that work cooperatively to monitor the environmental conditions, such as temperature, sound, pollutants, etc. They pas sata through the network to a base station and announce to the users.

In general, we usualy assumed that all nodes are cooperative and are willing to provide network services such as relaying data for others. However, this assumption is not strictly true in WSNs. Unlike traditional networks, sensor nodes in WSNs are limited in power, computational capacities, and memory. For some rational sensor nodes, they fix attention on saving their own resources and refuse to provide any service without their own interest for others. Such nodes are described as selfish nodes. Simulation based studies [2] show that even a small percentage (10-40\%) of selfish nodes can bring the network throughput considerably down (16-32\% degradation).

Clustering is widely adopted in WSNs, which divides the network into clusters and set cluster heads (CHs) responsible for data fusion. It has the advantages of low energy consumption, simple routing scheme and good scalability. For the nodes that serve as $\mathrm{CHs}$, their energy and resource consumption are normally faster than its member nodes due to the long-distance communication with the sink. Therefore a selfish node would refuse to declare itself as a $\mathrm{CH}$. 
Game theory [3] is a branch of mathematics that models situations where players (participants in a game) participate in a strategic situation. The selfishness of sensor nodes can be modeled by game theory. More specifically, the mechanism designs a game such that selfish behavior of the nodes induces a predictable strategy profile, and the output function for this predicted strategy corresponds to a Nash Equilibrium outcome. Namely, no node has an incentive to unilaterally deviate from this dominant strategy.

In addition, the communication link between a $\mathrm{CH}$ and the sink is of vital importance. Any disconnection of these links at certain point could result in a lost of all sensed data in its cluster region. A possible solution is to adopt data replication [4]. The idea is to keep copies of data in more nodes, so that if any failure occurs to the node that owns the original data, its information is not lost and can be retrieved through its copies. Here, we simply replicate the data in a $\mathrm{CH}$ to another node. So even network division occurs and separate the connection from $\mathrm{CH}$ to the sink, we can still have another node to send the cluster's data.

In this paper, we use a game-theoretic analysis to find conditions that-would make cooperation a preferred choice for the nodes in WSNs that had been elected as CHIs. And we address the potential problem of connectivity between a $\mathrm{CH}$ and the sink in the context of data replication.

The rest of the paper is organized as follows. Related work is discussed in section 2. In Section 3 we address $\mathrm{CH}$ election on the basis of game theory and adopt data replication in case of possible disconnection between an original $\mathrm{CH}$ and the sink. Performance evaluation is given in Section 4 and Section 5 concludes this paper.

\section{Related Work}

Clustering in wireless sensor networks is a hot topic. Various well known clustering routing protocol have been proposed, such as LEACH [5] and PEGASIS [6]. Energy consumption and communication latency are reduced, however, many assumptions do exist in such approaches. For example, nodes should have much information about other nodes, which is not practical in eality. Besides, most clustering protocols do not consider the selfishness of nodes

Selfishness in wireless network is a popular study. Incentive mechanisms have been proposed. In Tit-For-Tat (TFT) [7], the player cooperates on the first stage and does what its opponent did in the previous stage. However, a perceived defection may be unjustly punished due to packet collisions. GTFT [8] improves TFT by providing a tolerance threshold. Limited number of defections will not be punished. Ref. [9] studies the impact of packet collisions on the emergence of cooperation and proposed two schemes called OT and GT for milder conditions. They are theoretically effective, but practically unstable.

Various game-theoretic models have been adopted. Ref. [10] ensures that nodes reveal private information truthfully. However, it only focuses on the nodes' energy reporting strategy and fails to analysis other cooperating behaviors of the nodes. Ref. [11] provides a utility function to stimulate cooperate and study Nash Equilibrium theoretically. However, simalation remains to be implemented. GTEBR [12] solves the problem of uneven energy consumption as a sort of static game with so-called confidence probability. It depends on central control, which makes it not suitable for distributed autonomous environment. The balance of payment and cost is also hard to keep. Ref. [13] proposes a repeated-game model based on local detection and punishment mechanism of isolation, which takes account of the selfish nodes' future payoff expectations and their long-term desires for payoff. However, the proposed model is not suitable for WSNs as sensors nodes have constraint on energy. Ref. [14] designs a payoff function on path reliability and energy consumption. Using the punishment 
mechanism, the repeated game model can propel a Nash Equilibrium and decrease the defection possibility of selfish nodes. But it fails to consider effect of the distance between nodes on energy consumption. Ref. [15] defines a Trust Degree and Most Trustworthy Path (MTP) in consideration of the basic trust elements in WSNs such as security, energy constraint and routing reliability. An incentive and punishment scheme is proposed to resolve selfish nodes. Ref. [16] aims at solving the problem of retaliation situations after a node is falsely perceived as selfish to help restore cooperation quickly. This scheme is collusionresistant and can achieve full cooperation among nodes. However, it is based on the assumption that nodes share perceived dropping probability with each other truthfully. Ref. [17] combines a modified version of existed routing protocol with coalitional game theory in order to find the cheapest route in a group with respect to power consumption. How to choose corresponding leaders is not mentioned though. It just focuses on logic rewriting of the algorithm. Ref. [18] determines the route with least energy consumption and maximum cooperation among nodes via a game-theoretic approach. According to the payoff matrix, nodes are encouraged to participate with its best possible action,

Data replication is very effective for preventing deterioration of data aceessibility due to network division in wireless networks. Ref. [19] proposes data replication schemes in ad hoc networks. These schemes are based on the intuition that to improve data accessibility, replicating the same data near neighboring nodes should be avolded. In SAF, the access frequency for certain data is the major concern to decide which node should get the replica. DAFN pays extra attention to its connectivity with the neighbors, and DCG sets nodes into groups for later discussion. A later study $[20]$ extends the above methods by considering a more real environment with periodic dateupdate. In Ref. [21], each node belongs to certain cluster in which the probability of path availability can be bounded. Nodes exchange information with stable neighbors. Ref. [22] proposes some new schemes for data replication: Greedy-S considers both the size and access irequency of data; OTOO adopts a metric of combined access frequency which is related to the node and a neighbor of its own; RG sets groups for nodes that can share replica. Unlike the study of Hara, link failure probability and query delay are taken into consideration. Ref. [23] selects nodes as data replicas holders taking into account link bandwidth and remaining amount of batteries. Various parameters demand specific future study though.

\section{Our Proposed Game-theoretic Approach}

\subsection{Relevant models}

\subsubsection{Network model}

We assume that the network is composed of $N$ sensor nodes. They are uniformly dispersed within a circle field and continuously monitor their surrounding environment, as is shown in Figare 1. We also assume that one sink locates far away from the sensing region and receives data delivered by all the cluster heads. Moreover, the sink is mobile and changes its position in circle that ensures its average distance from every node is the same in a relative long-term run. Such setting reduces the energy hole problem to some extent. We make the following assumptions:

(1) All nodes are homogeneous and stationary after deployment.

(2) The multiple sink nodes are pre-located within the sensing field randomly.

(3) Nodes can adjust their transmission power according to the relative distance to receiver 
(4) Links are symmetric.

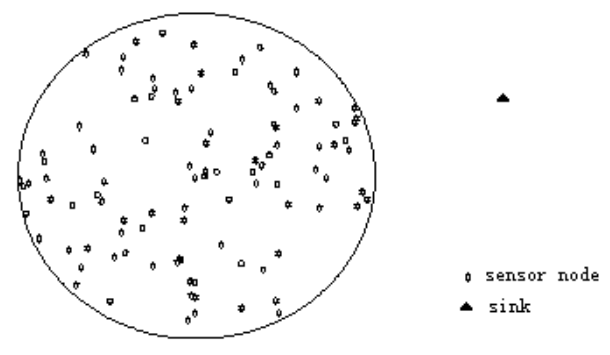

Figure 1. Network model

3.1.2. Energy model: We use similar energy model in [17]. Based on the distance between transmitter and receiver, a free space ( $d^{2}$ power loss) or multir path fading ( $d^{4}$ power loss) channel models are used.

Each sensor node will consume the following $E_{T x}$ amount of energy lo transmit a $\boldsymbol{l}$ bits packet over distance $\boldsymbol{d}$, where the $E_{\text {elec }}$ is the energy dissipated per bit to run the transmitter or receiver circuit, $\varepsilon_{f s}$ and $\varepsilon_{m p}$ represent the transmitter amplifier's efficiency and channel conditions:

$$
E_{T x}(l, d)=\left\{\begin{array}{l}
E_{\text {elo }}+l \varepsilon_{f s} d^{2}, d<d_{\infty} \\
l E_{\text {elec }}+l \varepsilon_{m p} d^{4}, d \geq d_{\infty}
\end{array}\right.
$$

To receive a packet, radio consumes enersy.

$$
\text { 2) } E_{R x}(l)=I_{E_{\text {elec }}}
$$

Cluster heads aggregate $n l$ bits data packets received from its members into a single $l$ bits fixed packet. The energy consumption is calculated as follows, where $E_{D A}$ is the data aggregation cost of a bit per signal:

$$
E_{\text {aggr }}(n, l)=n l E_{D A}
$$

\subsection{Selection of cluster heads}

In our stydy, the entire network is divided into $K$ equal clusters, as is shown in Figure 2 where $K=5$. Each cluster has one cluster head for data aggregation. Instead of direct communication with the sink, each member node in one cluster sends data to its $\mathrm{CH}$. Each $\mathrm{CH}$ receives the delivered data, makes aggregation and finally sends data to the sink far away. Such clustering method reduces the traffic load. Moreover, CHs locate in a more uniform way than the probabilistic deployed situation in LEACH. It prolongs the network lifetime and reduces the energy hole problem.

The selection of $\mathrm{CHs}$ is important. If a $\mathrm{CH}$ runs out of energy, all collected data in its cluster get lost and can no longer reach the sink. Therefore, we have the residual energy of a node stand out as a metric. Any node with the maximum residual energy in a 
cluster is chosen as a $\mathrm{CH}$. With $\mathrm{CH}$ roles change periodically, the network can survive for long time.

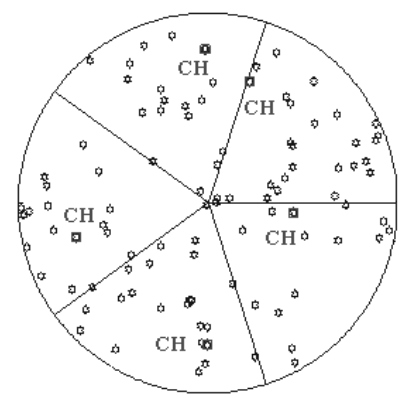

Figure 2. An example of $\mathrm{CH}$ selection

\subsection{Game-theoretic model for $\mathrm{CH}$ selection}

Since each cluster head consumes much energy and takes responsibility of sending data for cluster members, in the fact selfish nodes may refuse to declare itself as the $\mathrm{CH}$. They may tell lies about the value of its residual energy to avoid being selected.Hence the $\mathrm{CH}$ selection would fail to work. To solve the problem, we regard the $\mathrm{CH}$ declaration as a game and adopt a game-theoretic model to promote cooperation of selfish nodes.

We formally define the game as $G=\gtrless N, S, U>$, where $N(|N|=n)$ is the set of players, representing sensor nodes in the network $S=\left\{S_{i}\right\}$ is the set of available strategies; $U=\left\{U_{i}\right\}$ is the set of utility functions.

Players can either declare itself a $\mathrm{CH}$ or stay selfish by refusing to be the $\mathrm{CH}$. Letting $D$ be the strategy "declare myself as $\mathrm{CH}$ " and $R D$ be the strategy "refuse to declare as $\mathrm{CH}$ ", the strategy space is $S=\{$ Declare, Refuse to Declare $\}=\{D, R D\}$.

We define $c_{D}$ and $c_{R}$ respectively represent the cost of the node when it declares itself as $\mathrm{CH}$ and the refusing situation, shown in Equation (4) and (5) respectively. Here, $n_{\mathrm{CH}_{i}}$ stands for the number of nodes in cluster $\mathrm{CH}_{i}$.

$$
\begin{gathered}
C_{D} n_{C H_{i}} E_{R x}+E_{a g g r}+E_{T x\left(C H_{i}, \sin k\right)} \\
c_{R D}=E_{T x\left(\mathrm{~s}_{\mathrm{i}}, C H_{i}\right)}
\end{gathered}
$$

Role of cluster heads changes periodically. In the long term, $c_{D}$ and $c_{R D}$ can be regarded as constants or simplicity. Moreover, as the sink locates far away from the sensing region, the cost for delivering data to the sink is much larger than that to its $\mathrm{CH}$ according to the energy model, namely $c_{D}>c_{R D}$. In case nodes are reluctant to declare as $\mathrm{CH}$, a payoff $v$ is provided. From the perspective of an arbitrary node, as the equation (6) shows, if one node $i$ declares, the utility is $v-c_{D}$; if node $i$ refuses to be $\mathrm{CH}$ and luckily one other nodes in its cluster takes the responsibility by declaring itself as $\mathrm{CH}$, the utility of $i$ becomes $v-c_{R D}$; however, the worst condition is that neither the node itself nor any other node declares as $\mathrm{CH}$, therefore the player will be unable to send data towards the sink which leads to zero payoff in result. 


$$
U_{i}(\mathrm{~S})=\left\{\begin{array}{cc}
v-c_{D} & \text { if } S_{i}=D \\
v-c_{R D} & \text { if } S_{i}=R D \text { and } \exists j \in N, \text { s.t. } S_{j} \in D \\
0 & \text { if } S_{j}=R D, \forall j \in N
\end{array}\right.
$$

According to the assumption, all nodes are homogeneous. So it is impossible for each node to find a best response to the strategy choices of its opponents. Namely no pure-strategy Nash Equilibrium exists in our game. However, if we assume that each player is allowed to choose its strategy randomly following a probability distribution, a mixed-strategy Nash Equilibrium can be found.

For each node, the possibility of declaring itself as $\mathrm{CH}$ (i.e. playing $D$ ) is set as $p$, and the probability of refusing to declare (i.e. playing $R D$ ) is $1-p$. The expected utility function of playing $D$ is obtained as $U_{D}=v-c_{D}$. The expected utility function of playing $R D$ is obtained as $U_{R D}=\left(v-c_{R D}\right) \cdot\left(1-(1-p)^{N-1}\right)$, which implies at least one other node plays $D$.

At the equilibrium, we have $U_{D}=U_{R D}$. By solving the expression, we have

$$
p=1-\left(\frac{c_{D}-c_{R D}}{v-c_{R D}}\right)^{\frac{1}{N-1}}
$$

Once the probability $p$ is properly set a mixed-strategy Nash Equilibrium exists. In this case, with $p$ predetermined for all nodes each node has a natural incentive to cooperate and make declaration as a $\mathrm{CH}$.

For an arbitrary node, calculate it ayerage utility of a node $\bar{U}$, we have

$$
\begin{aligned}
\bar{U} & =U_{D} \cdot p+U_{R D} \cdot(1-p) \\
& =\left(\left(-c_{D}\right) \cdot p+\left(v-c_{R D}\right)\left(1-(1-p)^{N-1}\right) \cdot(1-p)\right. \\
& =\left(v-c_{R D}\right)-\left(C_{D}-c_{R D}\right) p-\left(v-c_{R D}\right)(1-p)^{N}
\end{aligned}
$$

At the nix-strategy Nas Equilibrium, by substituting ${ }^{p}$ from equation (7), we have

$$
\text { U. } \overline{U_{N E}}=v-c_{D}
$$

Letting the derivative of $\bar{U}$ equal to zero, we can compute a $p^{*}$ that makes the maximum average utility $\overline{U_{\max }}$. Respectively, we have

$$
\begin{gathered}
p^{*}=1-\left(\frac{c_{D}-c_{R D}}{v-c_{R D}} \cdot \frac{1}{N}\right)^{\frac{1}{N-1}} \\
\overline{U_{\max }}=\left(v-c_{R D}\right)-\left(c_{D}-c_{R D}\right) p^{*}-\left(v-c_{R D}\right)\left(1-p^{*}\right)^{N} \\
=v-c_{D}+\left(c_{D}-c_{R D}\right)\left(1-\frac{1}{N}\right)\left(\frac{c_{D}-c_{R D}}{v-c_{R D}} \cdot \frac{1}{N}\right)^{\frac{1}{N-1}}
\end{gathered}
$$


Compare $\overline{U_{\max }}$ and $\overline{U_{N E}}$, we have

$$
\begin{aligned}
\overline{U_{\max }}-\overline{U_{N E}} & =v-c_{D}+\left(c_{D}-c_{R D}\right)\left(1-\frac{1}{N}\right)\left(\frac{c_{D}-c_{R D}}{v-c_{R D}} \cdot \frac{1}{N}\right)^{\frac{1}{N-1}}-\left(v-c_{D}\right) \\
& =\left(c_{D}-c_{R D}\right)\left(1-\frac{1}{N}\right)\left(\frac{c_{D}-c_{R D}}{v-c_{R D}} \cdot \frac{1}{N}\right)^{\frac{1}{N-1}}
\end{aligned}
$$

According to Equation (12), when the number of nodes $\mathrm{N}$ increases and tends to infinity, namely $N \rightarrow \infty$, we have $\lim \left(\overline{U_{\max }}-\overline{U_{N E}}\right)=0$. That means the utility at Nash Equilibrium is almost equal to the maximum possible value.

\subsection{Data replication and selection of candidate $\mathrm{CHs}$}

Cluster heads receive all collected data from its cluster members and send the aggregated data to the sink. Cluster heads serve as bridges that conneet the sink and certain sensed area. They play a significant role. Therefore, once part of the hetwork becomes unstable and by chance it causes a disconnection of the link between certain $\mathrm{CH}$ and the sink, all data in the cluster would be lost. To solve this problem, we take measuresvia data replication.

During an update period, we set another sensor node despite the current $\mathrm{CH}$ as a candidate $\mathrm{CH}$. This candidate node replicates $\mathrm{CH}$ 's data. Thus when the sink fails to communicate with the $\mathrm{CH}$ at one point, it can still get a replica of cata from another node. Robustness of the network is improved due to such atata replication To encourage all member nodes to complete for the role of a candidate $\mathrm{CH}$, angextra payoff is provided if it has direct communication to the sink.

In one cluster, we adopl game-theoretre method to select a candidate $\mathrm{CH}$. All member nodes despite the $\mathrm{CH}$ are players. They have the desire of turning into a candidate $\mathrm{CH}$ in order to win the possible payoff. Ne assume that every player has its own valuation, and they bid against each other to win the game. In a wireless sensor network, we assume that all data collected by sensor nodes is periodically updated. Accordingly, their corresponding CHs update data.

For example, in a cluster $k$, we have $p_{k}^{i}$ represent the probability that a member node $i$ performs an access request for the data in $\mathrm{CH}$ within a unit of time; $T_{k}$ stands for the update period of its $\mathrm{CH}, t_{k}$ denotes the time that has passed for all member nodes to complete its data delivery, $\tau_{k}$, the difference between $T_{k}$ and $t_{k}$, represents the time that it provides for menber nodes to access data in $\mathrm{CH}$. For a member node $i$, we have the access frequency $r_{k}^{i}$ :

$$
r_{k}^{i}=\frac{p_{k}^{i} \cdot \tau_{k}}{T_{k}}=\frac{p_{k}^{i} \cdot\left(T_{k}-t_{k}\right)}{T_{k}}
$$


Let $R_{k}^{i}$ represent the cost for $i$ to replicate data in its $\mathrm{CH}$. It is related to both its data access frequency $r_{k}^{i}$ and a transmission cost to its $\mathrm{CH}$ that is denoted as $c_{k}^{i}$. We have

$$
R_{k}^{i}=r_{k}^{i} \cdot \mathrm{c}_{k}^{i}
$$

The focal point of data replication is to minimize total cost in the network. It not only includes its cost for data replication. In fact, once a member node $i$ is selected as the candidate $\mathrm{CH}$, it now has the possibility of communicating with the sink. Such communication cost $c_{\sin k}^{i}$ should also be taken into consideration. Moreover, due to extra burden of communication cost, there is chance that it consumes all its energy becones invalid, and in result causes the energy hole problem. To alleviate the problem, its residual energy $E_{\text {residual }}{ }^{i}$ becomes an essential factor. We aim to find the proper candidate with not only less cost for data replication, but also larger residual energy and less communication cost with the sink. Players offer bids. The one with the highest value is elected as the winner and get selected as the candidate $\mathrm{CH}$. For node $i$, we have its bid $B_{k}^{i}$ defined as follows:

$$
B_{k}^{i}=\frac{E_{\text {residual }}^{i}}{R_{k}^{i} \cdot \mathrm{c}_{\sin k}^{i}}=\frac{E_{\text {residual }}^{i}}{r_{k}^{i} \cdot \mathrm{c}_{k}^{i} \cdot \mathrm{c}_{\sin k}^{i}}=\frac{E_{\text {residuat }}}{p_{k}^{i} \cdot \theta^{i} \cdot \mathrm{c}_{\mathrm{sin} k}^{i}}
$$

According to all member nodes in the same cluster, they share the same value of $T_{k}$ and $t_{k}$, and namely the same $\frac{T_{k}}{\left(T_{k}-t_{k}\right)}$. Let $\alpha=\frac{T_{k}}{\left(T_{k}-t\right)}$ and $r$ gard $\alpha$ as a constant. We simplify the bid.

$$
B_{k}^{i}=\alpha \cdot \frac{E_{\text {residual }}^{i}}{B_{k}^{i} \cdot \mathrm{c}_{k}^{i} \cdot \mathrm{c}_{\mathrm{sin} k}^{i}}
$$

For any player in the game of data replication, its bit remains the private information and can not be known by any other players. As every player has its own valuation and whether or not one wants to be the pumer depends only on the price he will have to pay, namely the bid in normal auction. Therefore, instead of submitting its real valuation, nodes may have a tendency to perform speculation by offering a higher value. Thus they may win the game with actually less payment.

Such sifuation is suitable for the adoption of the second price sealed auction, which is also known as "the Vickrey auction". It was proposed by William Vickrey in 1961[19]. Such auction can suppress the potential speculation of any player and in the case of asymmetric information the outcome of the game can reach Pareto Optimality [3]. It stands for an ideal state of the resource allocation where no other outcome can make at least one player strictly better off on the premise that other players maintain well off. That is, a Pareto Optimal outcome cannot be improved upon without hurting at least one player.

In a second price sealed auction, every player submits a bid without the knowledge of others' information. It is suitable for the data replication procedure as an incomplete information game. The one with the highest bid wins and only needs to pay a price equal to 
the second highest bid. And here the payoff provided according to the incentive provided for a $\mathrm{CH}$.

Regardless of its bid, the price a player would have to pay is determined only by the bids of the others. The higher his bid is, the greater the possibility of winning becomes. But if his bid is higher than its own true valuation, once the highest bid of others (i.e. the current second highest bid) is also larger than its valuation, what he has to pay is more than the valuation. It is unworthy. Therefore, bidding one's true valuation is the best choice. Any unilateral departure from such action can not bring any more payoffs which meets the case of Nash equilibrium. As rational nodes, all players should tell the truth by bidding his true valuation. The adoption of the second price sealed auction can efficiently achieve load balancing, rational allocation of resources, and the optimal control of network traffic.

\section{Performance Evaluation}

Simulate a wireless sensor network using NS2. We have" sensor nodes uniformly distributed in a $500 \times 500$ square region and the sink located far away, as it is shown in Figure 3.

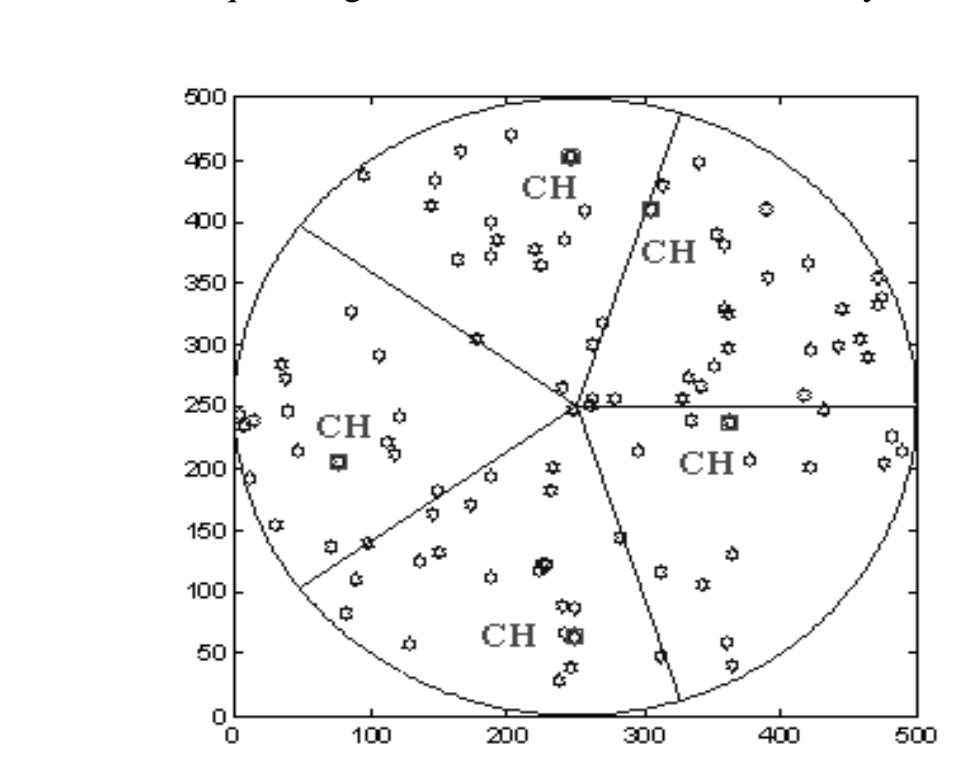

\section{Figure 3. Network for simulation}

Here, we have a simulation time of 60 seconds. And for simplicity, we can study one of the clusters - The proposed payoff for a $\mathrm{CH}$ is represented as a later priority in data delivery. For example. when a member node A and another node B both send data to the sink via their current cluster head at sometime, if $\mathrm{A}$ used to be the $\mathrm{CH}$, we have $\mathrm{A}$ send its data first and let $\mathrm{B}$ wait for a while till it gets turns to transmit its data. 


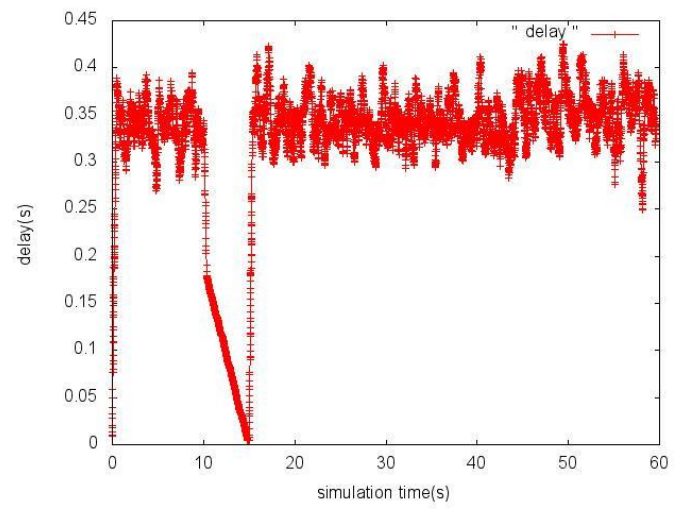

Figure 4. Data delay for a certain node

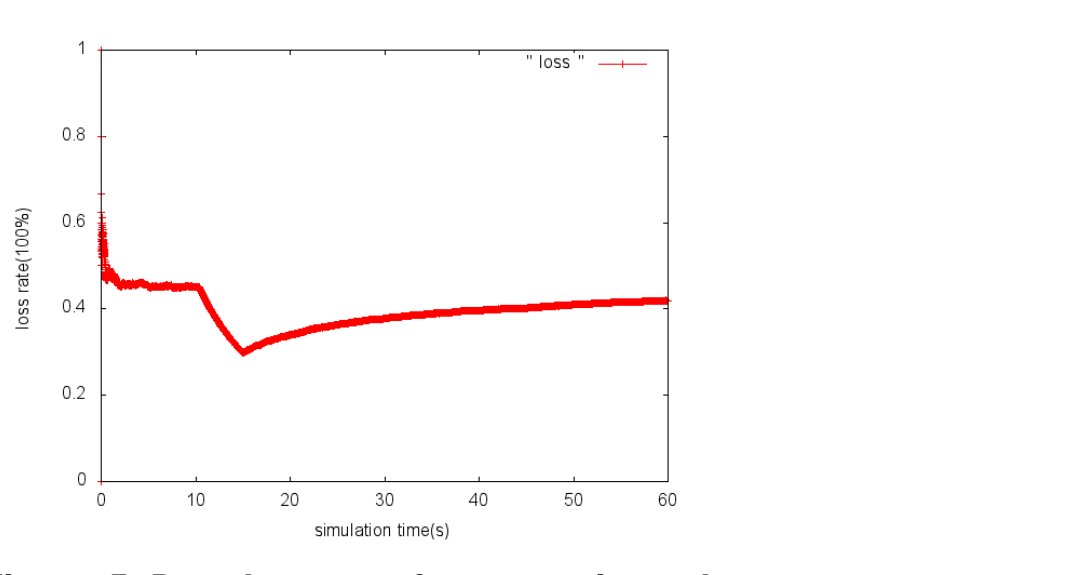

Figure 5. Data loss rate for a certain node

Figure 4 and Figure 5 Shows the changes in the aspect of delay and loss rate for a node that is once chosen as the cluster head respectively. We assume that it is selfish and refuses to be $\mathrm{CH}$ from the beginning but changes to cooperate during the $10^{\text {th }}$ to $15^{\text {th }}$ second. From the figures, It is obvious that or game-theoretic mechanism can result in a reduction the transmission delay for data and provide a relative lower data loss rate. Therefore, it becomes a rational choice for a node to deviate from selfishness.

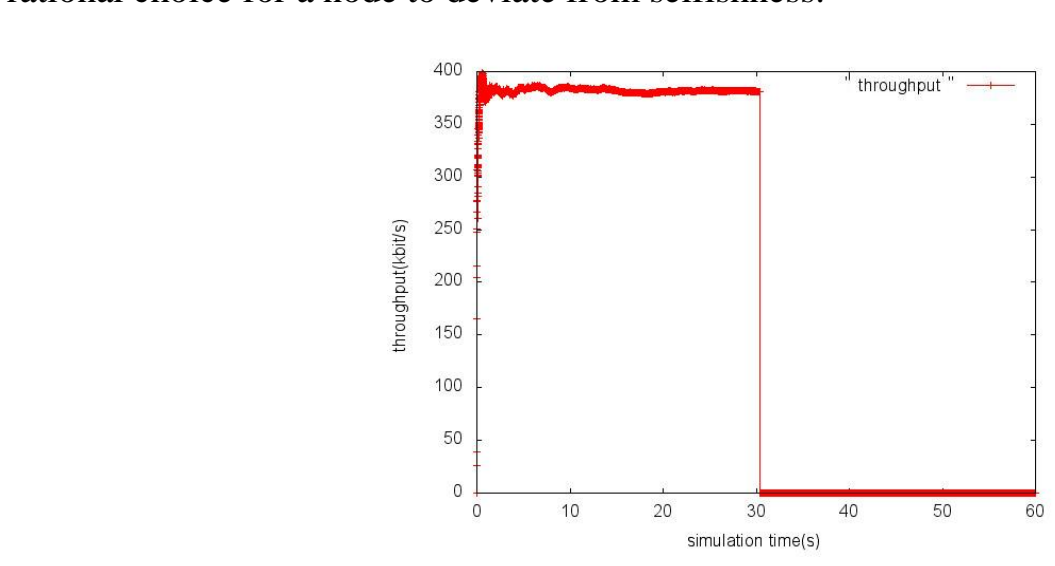

Figure 6. Throughput of a sink related to a cluster's data (no data replication used) 


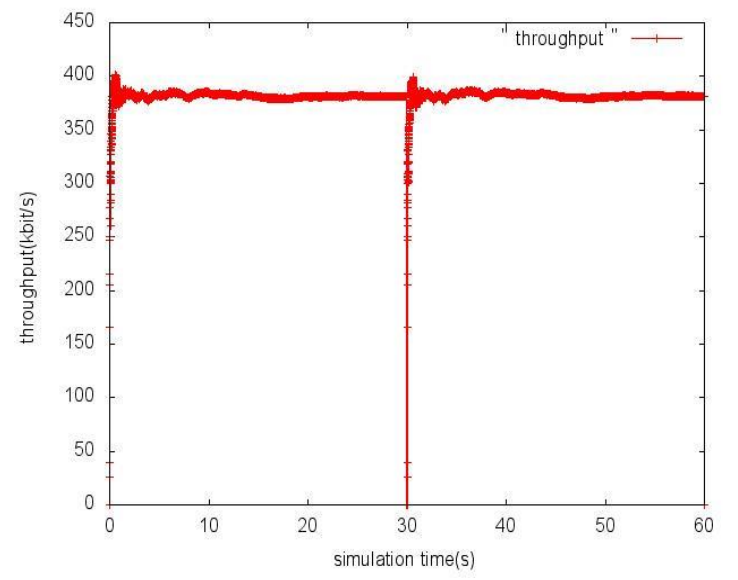

Figure 7. Throughput of a sink related to a cluster's data (data replication used)

Moreover, we study the throughput for the sink that receive the collected data from the chosen cluster. If the connection between its $\mathrm{CH}$ and thesink breaks up, as it is shown in Figure 6 that a link disconnection occurs at $30^{\text {th }}$ second the throughput of the sink drops to zero. However, with data replication, the sink is able to get the chuster' data from the replica in the candidate $\mathrm{CH}$. Figure 7 shows that the throughput of the sink for the cluster's data is barely changed.

\section{Conclusions}

Clustering is an efficient method in wireless sensor networks. Essential operations in clustering include the selection of $\mathrm{CHs}$, which have much responsibility for data delivery. However, due to theirlimited resources, nodes have selfishness which may affect the efficiency of clustering. In this paper, game theory is adopted to encourage nodes to serve as a $\mathrm{CH}$ Moreover, candidate $\mathrm{CH}$ s are selected that replicates the data in $\mathrm{CH}$ under the circumstance of a second price sealed auction. Such data replication reduces the risk of disconnection betyeen a $\mathrm{CH}$ and the sink. Simulation results prove that our game-theoretic clustering approach provides good performance.

\section{Acknowledgements}

This work was supported by Jiangsu Province Universities Natural Science Research Program (NO.11KJB510010 and Jiangsu Province Research and Innovation Project for College Graduates (NO.CXZZ12_0515) It was also supported by the Industrial Strategic Technology Development Program (1004 740) funded by the Ministry of Trade, Industry and Energy (MOTIE Korea), and by the Natural Science Foundation of Jiangsu Province (No. BK2012461). Professor Jeong-Uk Kim is the correspending author.

References

[1] I. Akyildiz, W. Su, Y. Sankarasubramaniam and E. Cayirci, (Eds.), IEEE Commun, Mag., vol. 8, (2002), pp. 102.

[2] P. Michiardi and R. Molva, "Simulation-based Analysis of Security Exposures in Mobile Ad Hoc Networks", Proceedings of the European Wireless Conference, (2002).

[3] D. Fudenberg and J. Tirole, "Game Theory", MIT Press, (1991).

[4] V. R. Q. Leithardt, C. Geyer, J. Sá Silva and R. Silva, "Use of Data Replication in WSNs", Proceedings of the IEEE International Conference on Communications Workshops (ICC), Brazil, vol. 1, (2010). 
[5] W. Heinzelman, A. Chandrakasan and H. Balakrishnan, "Energy-efficient Routing Protocols for Wireless Microsensor Networks", Proceedings of the 33rd Annual Hawaii International Conference of System Sciences, Maui, Hawaii, (2000) January 4-7.

[6] S. Lindsey and C. S. Raghavendra, "PEGASIS: power-efficient gathering in sensor information systems", Proceedings of the Int'l Conf. Comm. (ICC '01), (2001).

[7] R. Axelrod, "The Emergence of Cooperation among Egoists", The American Political Science Review, (1981), pp. 75, 306.

[8] J. Wu and R. Axelrod, "How to Cope with Noise in the Iterated Prisoner's Dilemma", The Journal of Conflict Resolution, vol. 39, (1995), pp. 183.

[9] F. Milan, J. J. Jaramillo and R. Srikant, "Achieving cooperation in multichip wireless networks of selfish nodes", Proceedings of the Game Theory for Networks Pisa, Italy, (2006).

[10] W. D. Wang and Q. X. Zhu, "A hierarchical clustering algorithm and cooperation analysis for wireless sensor networks", Journal of Software, vol. 17, no. 1157, (2006).

[11] L. Li, S. S. Dong and X. M. Wen, "The Analyses of Cooperation Mechanism Based on Game Theory in Wireless Ad hoc Network", Journal of Electronics \& Information Technology, vol. 26, no. 1329, (2007).

[12] J. Zeng and C. D. Mu, "Game Theory-based Energy Balance Routing with Incomplete Information in Wireless Sensor Networks", Acta Automatica Sinica, vol. 34, no. 317, (2008).

[13] Y. Lu, J. Shi and L. Xie, "Repeated-game Modeling of Cooperation Enforcement in Wireless Ad Hoc Network", Journal of Software, vol. 19, no. 755 (2008).

[14] Q. Liu, P. Xian and S. T. Guo, "Repeated-Game Theory of Cooperative Model in Wireless Sensor Network Routing", Chinese Journal of Sensors and Actuators, vol. 23, no. 1322, (2010).

[15] J. T. Jiang, Z. G. Chen and X. H. Deng, "Trustworthy Path Research in WSN Based on Dynamic Complete Information Game", Journal of Chinese Computer Systems, vol. 31, no. 1478. (2010).

[16] J. J. Jaramillo and R. Srikant, "A game theory based reputation mechanism to incentivize cooperation in wireless ad hoc networks", Ad Hoc Networks, vol. 8, n0.416, (2010).

[17] F. Kazemeyni, E. B. Johnsen, O. Owe and I. Balasingha, "Grouping Nodes in Wireless Sensor Networks Using Coalitional Game Theory", Proceedings of the 16th IEEE International Conference on Engineering of Complex Computer Systems (ICECCS), (2011).

[18] J. Gupta, I. Kumar and A. Kacholiya, "Game Theoretic Approach to Resolve Energy Conflicts in Ad-Hoc Networks", Advances in Computing and Communications, vol. 193, (2011), pp. 205.

[19] T. Hara, "Effective replica allocation in ad hoc networks for improving data accessibility", Proceedings of the 20th annual joint conference of the IEEE computer and communications societies, (2001), pp. 1568.

[20] M. Shinohara, T. Hara and S. Nishro, "Replica-Allocation Considering Power Consumption in Mobile Ad Hoc Networks", Proceedings of the 4th Annwal IEEE International Conference on Pervasive Computing and Communications Workshops, (2006).

[21] J. Zheng, X. C. Lu and Y. J. Wang, "A Clustering-based Data Replication Algorithm in Mobile Ad Hoc Networks for Improving Data Availability", Journal of Software, vol. 16, (2005), pp. 1474.

[22] Y. Zhang, L. Yin J. Zhao and G. Cao, Balancing the Tradeoffs between Query Delay and Data Availability in Ad Hoc Networks", IEEE Transaction on Parallel and Distributed Systems, vol. 8, (2011).

[23] W. Z. Lu, Y.C.Zhou, J. H. Li and B. P. Yan, "A Hierarchical Data Replication Method in Ad Hoc Networks", Proceedings of the 2010 2nd International Conference on Computer Engineering and Technology (ICCET), vol. 23, (2010).

[24] M. Shinohara and T Hara, S. Nishio, "Replica Allocation Considering Power Consumption in Mobile Ad Hoc Networks", Proceedings of the 4th Annual IEEE International Conference on Pervasive Computing and Communications Workshops, (2006).

[25] T. Rappaport, "Wireless Communications: Principles \& Practice, Englewood Cliffs", Prentice-Hall, (1996).

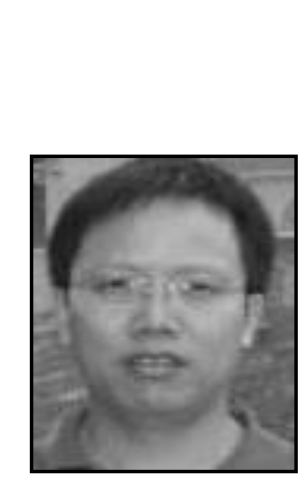

\section{Authors}

\section{Zhanyang Xu}

He obtained his Masters in Computer Engineering from the School of Computer Science and Technology, China University of Mining and Technology (CUMT) in 2004. He graduated as the top student in the Nanjing University of Posts and Telecommunications (NJUPT) where would obtain his PhD in 2012. At the same time, he is serving as a full time faculty in the School of Computer \& Software, Nanjing University of Information Science \& Technology 
(NUIST). His research interest includes wireless communication network, wireless sensor networks and Internet of things.

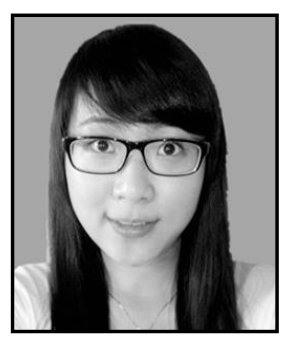

\section{Yue Yin}

She received the Bachelor degree in Applied Computing from Nanjing University of Information and Science Technology in 2011. She is currently pursuing a Master degree in Technology of Computer Application in the former institution. Her research interests include wireless sensor networks and Internet of things, specifically in fields of algorithm improvement and data replication.

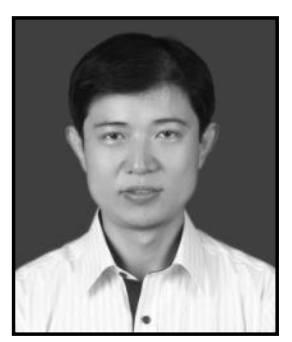

\section{Jin Wang}

He received the B.S. and M.S. degree in the Electron cal Engineering from Nanjing University of Posts and Telecommunications, China in 2002 and 2005, respectively. He received PhD. degree in the Ubiquitous Computing laboratory from the Conputer Engineering Department of Kyung Hee University Korea in 2010. Now, he is a professor in the Computer and Software Institute, Nanjing University of Information Science and Technology. His research interests mainly include routing protocol and algorithm design, performance evaluation and optimization for wireless ad hoc and sensor networks. He is a member of the IEEE and ACM.

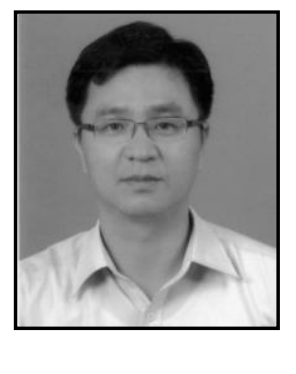

\section{Jeong-Uk Kim}

He obtained his BS. degree in Control and Instrumentation Engineening from Séoul National University in 1987, M.S. and Ph.D. degees in Electrical Engineering from Korea Advanced Institute of Scrence and Technology in 1989, and 1993, respectively. He is a professor in Sangmyung University in Seoul. His research interests include smart grid demand response, building automation system, and renevable energy.

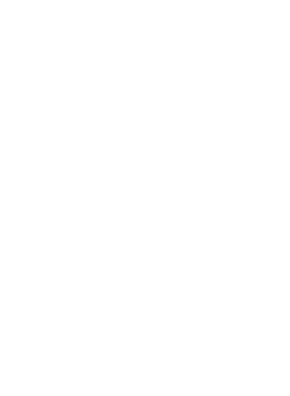<smiles>[Li]</smiles> 
International Journal of Hybrid Information Technology

Vol.7, No.1 (2014)

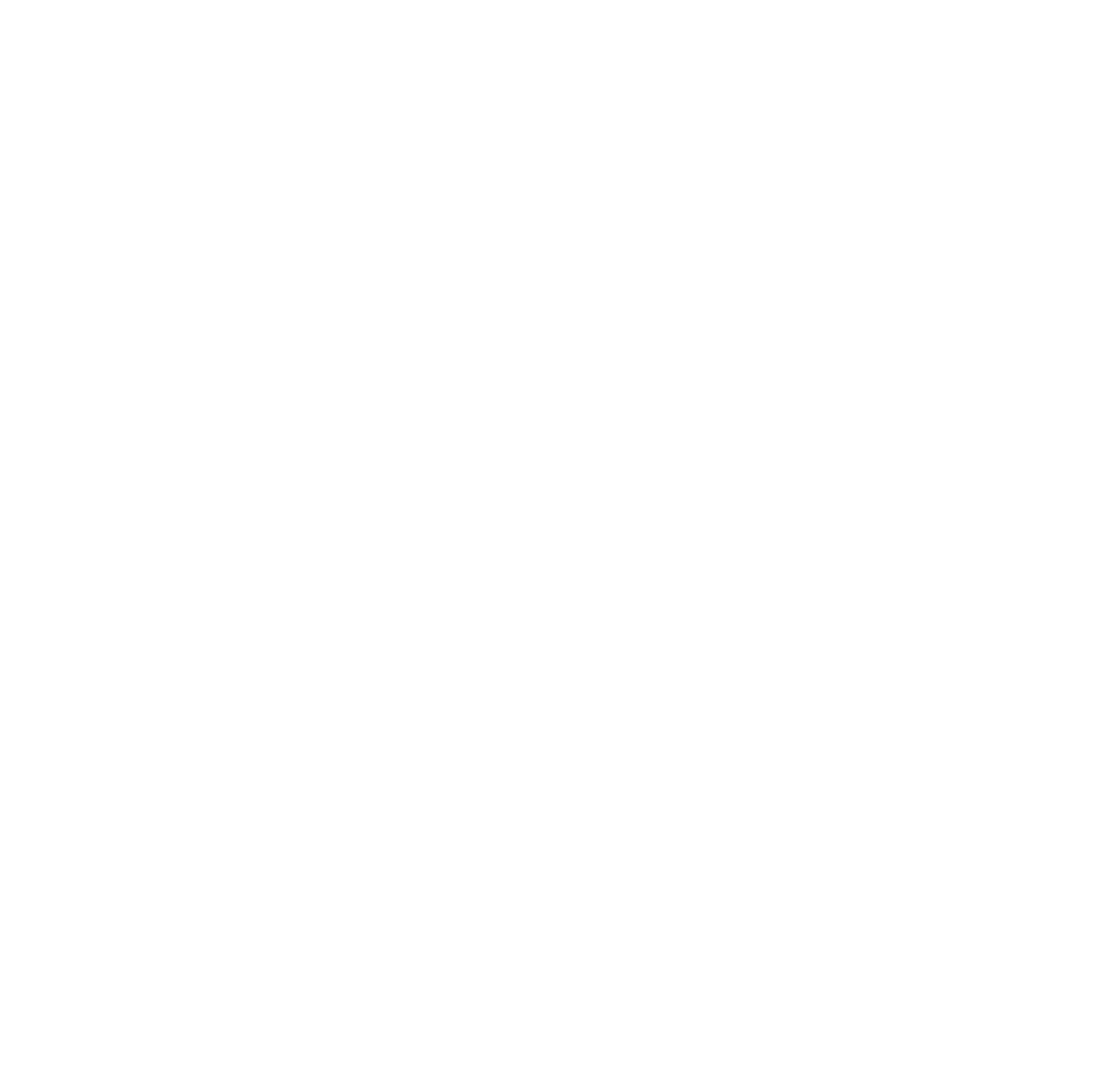

